\title{
Use of Chinese Herbal Medicine as an Adjuvant for Cancer Treatment: A Randomized Controlled Dose-Finding Clinical Trial on Lung Cancer Patients
}

\author{
King-Fai Cheng, Ping-Chung Leung* \\ Institute of Chinese Medicine, The Chinese University of Hong Kong, Hong Kong, China. \\ Email: pingcleung@cuhk.edu.hk
}

Received December 22 ${ }^{\text {nd }}, 2010$; revised April 10 ${ }^{\text {th }}$, 2011; accepted April 25 ${ }^{\text {th }}, 2011$.

\begin{abstract}
Background: Cancer has become a leading cause of death world-wide. In Hong Kong, cancer accounted for $24.8 \%$ of deaths in 1980, rising to 31.3\% in 1998. The conventional treatment of cancer usually includes surgery, radiotherapy and chemotherapy. These conventional therapies do not guarantee not relapse and are often associated with serious side effects. Using Chinese Medicine (CM) as an adjunctive treatment is commonly practiced in Chinese Communities to support patients being treated with conventional modern medicine, with the aim of alleviating the side effects, and improving self-defense and their quality of life. Well-designed and conducted clinical trials could give evidence of the efficacy of CM. This study investigated the clinical efficacy through the well designed clinical trial, and the implementation of carrying out the trial, to assess the adjuvant and supportive effect on lung cancer patients. Methods: The clinical study was designed as a randomized controlled trial to investigate the dose-dependent effects. Primary endpoint was the difference of Functional Assessment of Cancer Therapy-General (FACT-G) score. Results: 41 eligible subjects were enrolled and randomly divided into 2 groups, 21 in high dose group and 20 in low dose group. Sub-domains of PWB (Physical well-being) and EWB (Emotional well-being) as well as FACT-G total score were significantly improved in high dose group when compared with low dose group after 12 weeks treatment $(p=0.015,0.006$ and 0.012 , respectively). Conclusions: Holistic approach using quality of life as parameters to evaluate the efficacy of CM is an important compromise. Well-designed clinical trial can provide convincing evidence to support CM's efficacy. The study demonstrated that quality of life of patients with lung cancer could be beneficial from the supportive care with $C M$.
\end{abstract}

Keywords: Lung Cancer, Quality of Life, Chinese Medicine, Randomized Controlled Trial, Supportive Care

\section{Introduction}

The incidence of cancer has increased significantly all over the world in the last ten years and has become a major concern of health care systems [1,2]. In Hong Kong for instance, cancer has become the leading cause of death, accounting for $24.8 \%$ in 1980 , rising to $31.3 \%$ in 1998 [1]. The crude mortality rate of lung cancer was 206.5 per 100,000 in men and 126.8 in women for 1999 [1] and there has been an increasing trend in the incidence [3]. In China, according to the second national retrospective mortality survey conducted during 19901992, cancer had become the second most common cause of death [4-7]. In between, lung cancer is one of the leading causes of cancer deaths globally. It carries a greater mortality than colorectal, breast and prostate cancers collectively. Survival has not significantly improved in spite of newer therapies. For many centuries, Asian communities have used traditional medicines to treat cancer. Ginseng, for example which has recently been found to help preventing cancer has been widely used [8]. Chinese medicinal herbs are being investigated when used as adjuvant therapeutic agents for the patients who are undertaking or after conventional treatment [9-12]. In view of the high-symptom burden and severe morbidity, evaluation of quality of life (QOL) becomes important in these patients. QOL is now considered to be 
an essential component of lung cancer management and should be assessed routinely. In spite of several advancements in the chemotherapeutic regimens and the addition of many newer drugs, the 5-year survival has improved only marginally from $5 \%$ in the 1950 s to approximately $14 \%$ by 1996 [13]. In such a grim scenario, the evaluation and improvement of quality of life (QOL) as well as alleviation of symptom distress have assumed great importance in the overall management of these patients.

Clinical observations suggest that many traditional Chinese medical therapies may be effective for the supportive care of cancer patients [14,15]. Moreover there is an increase in the popularity of complementary / alternative medicine (CAM) for cancer patients in Europe and America and a prevalence of around $80 \%$ of the general population has been reported $[16,17]$. Among the CAM modalities, traditional Chinese medicine (TCM) is particularly favored because it is based on a well-established conceptual framework, albeit on a unique philosophical premise [18]. Unfortunately, the quality of the reported clinical trials using traditional Chinese medicine is usually poor, both in design, implementation and assessment of results [19-21].

As health services are becoming increasingly driven by the evidence-based approach, there is an urgent need for the evaluation of the efficacy and safety of the Chinese Medicine interventions through well-designed clinical trials. One cannot assume that CM treatments are all harmless, effective, and cost-effective. Adverse effects such as liver or renal damages have been reported, and the need for CM research on efficacy and safety has become undoubtedly clear. Adverse effects of herbs and drug-herb interactions should be carefully studied. Explorations on the active components, as well as the quality control of herbs should be seriously studied. To determine whether $\mathrm{CM}$ is a valuable adjuvant treatment to conventional therapy, we designed and conducted a ran- domized controlled clinical trial using Chinese herbal medicine as an adjuvant agent to evaluate its supportive effects on the patients with lung Cancer.

\section{Material and Method}

\subsection{Participants}

Forty-one lung cancer patients who had completed the conventional therapies were recruited and assessed for eligibility.

The inclusion criteria included a wide age range between 18 and 80 years, historically or cytologically confirmed lung cancer, Eastern Cooperative Oncology Group performance (ECOG) score of less than 2; life expectancy at least 12 months, completion of all conventional treatment and signing of an informed consent form. The subjects would be excluded if oncological emergencies discovered; taking of immunomodulative agents or herbal medicine within the past 2 weeks, and herbal hypersensitivity.

\subsection{Design of the Clinical Trial}

\subsubsection{Objectives}

The primary objective was to determine if a Chinese Medicine (CM) formula could improve the quality of life of patients with lung cancer.

\subsubsection{Design}

The clinical trial was designed as a randomized, dosedependent study to assess the efficacy and safety of the $\mathrm{CM}$ formula in patients suffering from lung cancer. The effectiveness of the CM was judged by the results of clinical trial that is designed and conducted in accordance with the principles of evidence-based medicine and randomized controlled trial (RCT) (Figure 1).

Randomization

Each patient eligible for the study was allocated to one of the two treatment groups according to a computer-generated randomization code list block of 10. After

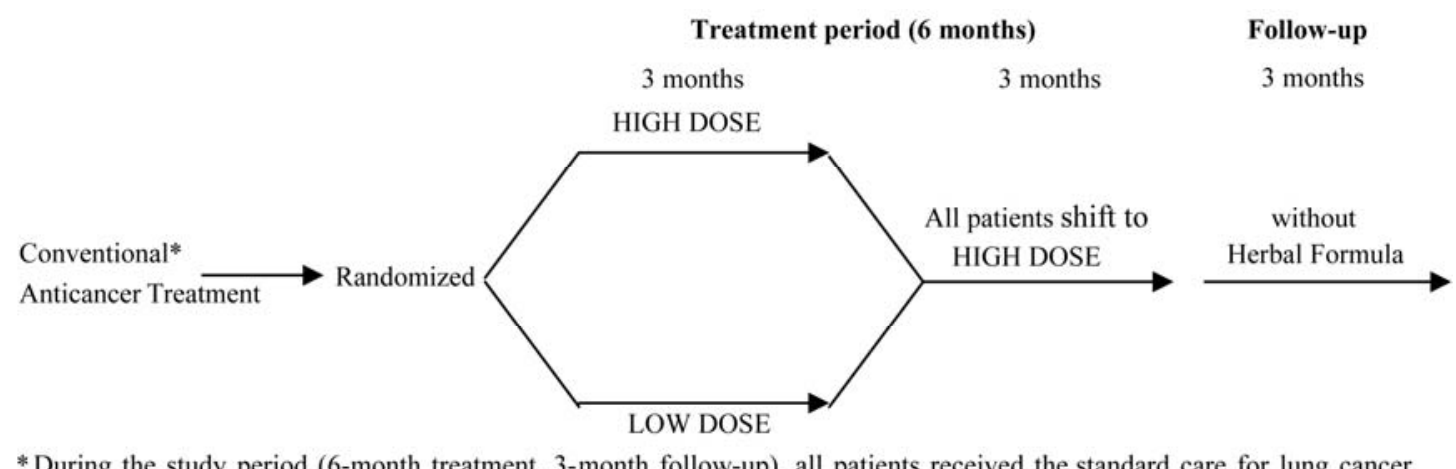

Figure 1. Design of clinical trial. 
the investigators confirmed that all inclusion criteria were fully met and that there were no reasons for exclusion, an eligible patient was given a patient number in the order of entry into the baseline study.

Blinding

The study was designed as a double-blind, randomized trial. Neither the investigators nor the participants knew which treatment was to be given [22]. The supplier of the $\mathrm{CM}$ ensured that the high dose and low dose matched well in their inner \& outer packaging. The CM preparation was a liquid product containing herbal extracts.

\subsubsection{Endpoints}

The primary outcome measures were Quality of Life as measured by means of Version 4 of the Functional Assessment of Cancer Therapy-General (FACT-G). The FACT-G was administered on the first day of treatment (baseline), week 12, week 24 and at the completion of the follow-up after treatment (week 36). The FACT-G, which is general questions relevant to all cancer patients, is a 27-item questionnaire consisting of four domains: physical, emotional, social and functional well-being. Each domain consists of six to seven questions scored by using a Likert-type scale ranging from 0 to 4 . The higher FACT-G score, the better is the patient's quality of life.

The Chinese Medicine preparation is used aimed at the strengthening of the whole body-mind system through the enhancement in resistance and tolerance through a holistic approach. The investigational drug is a product containing Ginseng, Wild Hawthorn, Jujube, Wu Wei Zi, Soybean etc. The preparation is believed to induce immune adjustment. The exact mechanism(s) through which this occurs is unknown. However, preliminary tests on mice revealed promising immunomodulating effects. These Chinese herbs had long been known for their pharmacological effects and/or their nutritional values. The toxicity of these herbs is considered to be low.

Secondary outcome measures included assessment of liver function and renal function.

\subsection{Schedule of Events}

Clinical evaluations included the medical history, assessment for ECOG performance status, laboratory tests and immune function tests, which were presented on Table 1 .

\subsection{Follow-up Evaluation}

It was essential to determine the health status of all patients entered into the trial, even those who did not return to the clinic for all follow-up appointments, with telephone calls or questionnaires. The duration of follow-up monitoring was the period of time after the intervention during which the study subjects were scheduled to be observed and evaluated in the follow-up period of 8 weeks (week 28 - 36).

\subsection{Statistical Considerations}

Comparability of LOW DOSE and HIGH DOSE groups at baseline were determined by using Student t-test and Chi-square test. For sex, age distributions and ECOG score Chi-square method was used. Student t-test was used for baseline height, weight and blood chemistry parameters. Baseline values for LFT and RFT were defined as the average of values taken at screening and

Table 1. Frequency for obtaining study parameters.

\begin{tabular}{|c|c|c|c|c|c|c|c|}
\hline \multirow[b]{2}{*}{ Event } & \multirow{2}{*}{$\begin{array}{c}\text { Pre-treatment } \\
\text { Screening }\end{array}$} & \multicolumn{5}{|c|}{ Treatment } & \multirow[t]{2}{*}{ Post-Treatmen } \\
\hline & & Baseline & & & & & \\
\hline Week & $-3-0$ & 0 & 4 & 8 & 12 & 24 & $28-36$ \\
\hline Visit & Vo & V1 & V2 & V3 & V4 & V5 & V6 \\
\hline Informed Consent & $\mathrm{X}$ & & & & & & \\
\hline Incl./Excl. Criteria & $\mathrm{X}$ & & & & & & \\
\hline Medical History & $\mathrm{X}$ & & & & & & \\
\hline Physical Exam & $\mathrm{X}$ & & & & $\mathrm{X}$ & $\mathrm{X}$ & $\mathrm{X}$ \\
\hline Vital Signs & $\mathrm{X}$ & & $\mathrm{X}$ & $\mathrm{X}$ & $\mathrm{X}$ & $\mathrm{X}$ & $\mathrm{X}$ \\
\hline Laboratory $^{\mathrm{a}}$ & $\mathrm{X}$ & $\mathrm{X}$ & & & $\mathrm{X}$ & $\mathrm{X}$ & $\mathrm{X}$ \\
\hline Quality of Life (FACT-G) & & $\mathrm{X}$ & & & $\mathrm{X}$ & $\mathrm{X}$ & $\mathrm{X}$ \\
\hline ECOG Score & & $\mathrm{X}$ & & & $\mathrm{X}$ & $\mathrm{X}$ & $\mathrm{X}$ \\
\hline Adverse Events & & & $\mathrm{X}$ & $\mathrm{X}$ & $\mathrm{X}$ & $\mathrm{X}$ & $\mathrm{X}$ \\
\hline Dispense Study Product & & $\mathrm{X}$ & $\mathrm{X}$ & $\mathrm{X}$ & $\mathrm{X}$ & & \\
\hline Dosage Record & & & $\mathrm{X}$ & $\mathrm{X}$ & $\mathrm{X}$ & $\mathrm{X}$ & \\
\hline Compliance & & & $\mathrm{X}$ & $\mathrm{X}$ & $\mathrm{X}$ & $\mathrm{X}$ & $\mathrm{X}$ \\
\hline
\end{tabular}

Remark:

a: Laboratory tests to be performed is LFT \& RFT*.

*LFT: Liver Function Test; RFT: Renal Function Test. 
baseline (Day 0).

Changes in LFT and RFT were compared between groups and pre- and post-treatment within groups by using the analysis of covariance (ANCOVA) or pair student t-test. Changes will be confirmed by using Wilcoxon rank-sum tests, analysis of covariance with the baseline value as a covariate, and analysis of the percentage change from baseline.

All $\mathrm{P}$ values were two-sided and $\alpha$ level of significance was set at 0.05 .

\section{Results}

\subsection{Patients}

Seventy-two patients were screened and thirty-one were found unsuitable. 41 patients satisfied the inclusion/ exclusion criteria and entered the study. There were 19 drop outs due to the restarting of chemotherapy, gastric pain, metastasis etc (Figure 2).

Demographic and clinical characteristics for the high dose, and the low dose groups were listed in Table 2. Baseline demographic and clinical characteristics were compatible between the two groups.

During the study period, all patients were evaluated in the health status and drug compliance. The total drug compliance was 95\% in the High dose group, $86 \%$ in the Low dose group. The drug compliance in the high dose group was shown better than that of the low dose group $(\mathrm{P}=0.029)$ (Table 3).

\subsection{Quality of Life (QoL) - FACT-G}

Forty-one patients completed the FACT-G. The FACT-G total score and subscale score means for the two groups were listed in Table 4. The scores of High Dose group were higher than LOW Dose group at 3-month and 6-month time point respectively. The differences of PWB, EWB and FACT-G total score between the two groups had reached statistically significant level.

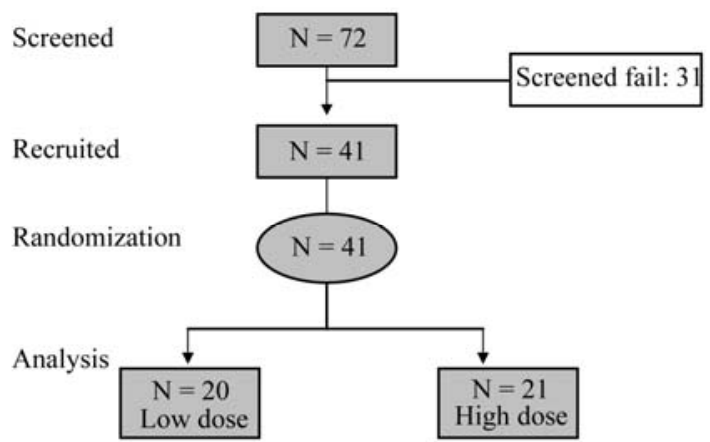

Figure 2. Flowchart for inclusion of patients.

Table 2. Baseline demographic and clinical characteristics.

\begin{tabular}{lccc}
\hline & Low dose & High dose & P value \\
\hline $\begin{array}{l}\text { No. of patients } \\
\text { Age }(y)\end{array}$ & 20 & 21 & \\
Gender & $58.3 \pm 11.6$ & $59.8 \pm 9.7$ & 0.663 \\
Male & & & \\
Female & $7(35 \%)$ & $11(52 \%)$ & 0.350 \\
& $13(65 \%)$ & $10(48 \%)$ &
\end{tabular}

ECOG score

$\begin{array}{lccc}0 \text { (normal activity) } & 7 & 8 & 0.547 \\ \begin{array}{l}\text { (Symptoms, but nearly } \\ \text { fully ambulatory) }\end{array} & 13 & 13 & \end{array}$

\begin{tabular}{lccc} 
Body weight (kg) & $56.4 \pm 10.8$ & $56.0 \pm 10.3$ & 0.903 \\
Blood pressure (mmHg) & & & \\
SYS & $134.2 \pm 21.0$ & $126.4 \pm 22.0$ & 0.252 \\
DIA & $79.3 \pm 21.9$ & $72.2 \pm 8.7$ & 0.177 \\
Respiration (rpm) & $20.8 \pm 2.0$ & $20.8 \pm 1.7$ & 0.987 \\
Pulse rate (beats/min) & $87.7 \pm 15.2$ & $80.4 \pm 16.4$ & 0.153 \\
\hline
\end{tabular}

Table 3. Average missing doses.

\begin{tabular}{cccccc}
\hline Group & Visit 2 & Visit 3 & Visit 4 & Visit 5 & $\begin{array}{c}\text { Total } \\
\text { compliance }\end{array}$ \\
\hline Low dose & 5 & 5 & 1 & 15 & $86 \%$ \\
High dose & 2 & 2 & 3 & 5 & $95 \%$ \\
P value & & & & & $\mathbf{0 . 0 2 9}$ \\
\hline
\end{tabular}

Table 4. Comparison of between-group mean scores and percent change (\%) from baseline at each visit.

\begin{tabular}{lcccccc}
\hline & \multicolumn{2}{c}{ Low Dose } & \multicolumn{2}{c}{ High Dose } & \multicolumn{2}{c}{ P value } \\
\cline { 2 - 5 } \cline { 6 - 8 } PWB & 3-month & 6-month & 3-month & 6-month & 3-month & 6-month \\
& $20.21 \pm 5.96$ & $21.00 \pm 6.01$ & $25.29 \pm 4.08$ & $25.31 \pm 2.72$ & $\mathbf{0 . 0 1 5}$ & $\mathbf{0 . 0 2 6}$ \\
SWB & $(-4.21)$ & $(-2.38)$ & $(4.27)$ & $(2.12)$ & & \\
& $20.60 \pm 4.57$ & $20.19 \pm 2.90$ & $21.67 \pm 3.90$ & $21.00 \pm 6.80$ & 0.520 & 0.687 \\
EWB & $(9.55)$ & $(-2.50)$ & $(10.11)$ & $(6.39)$ & & \\
& $16.62 \pm 3.10$ & $15.86 \pm 5.20$ & $20.50 \pm 3.61$ & $20.38 \pm 2.63$ & 0.006 & $\mathbf{0 . 0 0 9}$ \\
FWB & $(-3.32)$ & $(-5.59)$ & $(16.81)$ & $(16.66)$ & & \\
& $15.38 \pm 6.06$ & $16.93 \pm 5.62$ & $17.84 \pm 4.50$ & $18.23 \pm 6.37$ & 0.241 & 0.578 \\
FCAT-G total & $(-12.59)$ & $(-4.96)$ & $(1.14)$ & $(-4.50)$ & & \\
& $72.81 \pm 12.12$ & $73.98 \pm 13.92$ & $85.29 \pm 11.81$ & $84.92 \pm 13.80$ & $\mathbf{0 . 0 1 2}$ & $\mathbf{0 . 0 5 1}$ \\
\hline
\end{tabular}


Table 5. Physical well-being (PWB) subscale score and percent change (\%) from baseline.

\begin{tabular}{lllll}
\hline Group & \multicolumn{1}{c}{ Treatment } & Follow-up \\
\cline { 2 - 5 } & Baseline & Week 12 & Week 24 & Week 36 \\
Low dose & $22.80 \pm 4.91$ & $20.21 \pm 5.96(-4.21)$ & $21.00 \pm 6.01(-2.38)$ & $22.15 \pm 4.30(1.34)$ \\
High dose & $23.48 \pm 4.30$ & $25.29 \pm 4.08(4.27)$ & $25.31 \pm 2.72(2.12)$ & $24.22 \pm 4.18(-5.12)$ \\
P value & 0.641 & $\mathbf{0 . 0 1 5}$ & $\mathbf{0 . 0 2 6}$ & 0.275 \\
\hline
\end{tabular}

${ }^{*} \mathrm{P}$ values were calculated based on two-sample t tests comparing differences between-group means.

Table 6. Social/Family well-being (SWB) subscale score and percent change (\%) from baseline.

\begin{tabular}{lllll}
\hline \multirow{2}{*}{ Group } & \multicolumn{2}{c}{ Treatment } & Follow-up \\
\cline { 2 - 5 } & Baseline & Week 12 & Week 24 & Week 36 \\
\hline Low dose & $20.42 \pm 4.30$ & $20.60 \pm 4.57(9.55)$ & $20.19 \pm 2.90(-2.50)$ & $21.49 \pm 3.68(4.20)$ \\
High dose & $21.00 \pm 4.67$ & $21.67 \pm 3.90(10.11)$ & $21.00 \pm 6.80(6.39)$ & $21.37 \pm 5.65(10.37)$ \\
P value & 0.681 & 0.520 & 0.687 & 0.954 \\
\hline
\end{tabular}

${ }^{*} \mathrm{P}$ values were calculated based on two-sample t tests comparing differences in between-group means.

Table 7. Emotional well-being (EWB) subscale score and percent change (\%) from baseline.

\begin{tabular}{lllll}
\hline Group & \multicolumn{2}{c}{ Treatment } & Follow-up & Week 36 \\
\cline { 2 - 5 } & Baseline & Week 12 & Week 24 & $17.23 \pm 4.90(3.42)$ \\
Low dose & $18.30 \pm 4.01$ & $16.62 \pm 3.10(-3.32)$ & $15.86 \pm 5.20(-5.59)$ & $21.78 \pm 2.44(20.34)$ \\
High dose & $18.52 \pm 4.88$ & $20.50 \pm 3.61(16.81)$ & $20.38 \pm 2.63(16.66)$ & 0.019 \\
P value & 0.874 & $\mathbf{0 . 0 0 6}$ & $\mathbf{0 . 0 0 9}$ & \\
\hline
\end{tabular}

${ }^{*} \mathrm{P}$ values were calculated based on two-sample t tests comparing differences in between-group means.

As Table 4 shown, there was a significant improvement in PWB, EWB and FACT-G total score after 3-month treatment with the study CM. No significant differences were observed for the FACT-G social/family well-being (SWB) and functional well being (FWB) subscales.

Physical well-being measured by the FACT-G showed a significant improvement in the HIGH dose group compared with the LOW dose group at week 12 and week 24 ( $p=0.015$ and 0.026 respectively). When compared with the baseline, PWB score of the high dose group was increased $2.12 \%$ at the end of treatment (Week 24); however, in the low dose group there was a $2.38 \%$ decrease (Table 5, Figure 3).

No significant differences were observed for the FACT-G Social/Family well-being (SWB) between the two groups. When compared with baseline, SWB score of the high dose group was increased $6.39 \%$ at the end of treatment (Week 24); however, in the low dose group there was a $2.50 \%$ decrease (Table 6).

Emotional well-being (EWB) measured by the FACT$G$ showed a significant improvement for High dose compared with Low dose group at week 12, week 24 and follow-up visit ( $p=0.006,0.009$ and 0.019 respectively) When compared with baseline, EWB score of high dose group was increased $16.66 \%$ at the end of treatment (Week 24); however, in low dose group there was a 5.59\% decrease (Table 7, Figure 4).

No significant differences were observed for the FACT-G Functional well-being (FWB) between the two groups (Table 8).

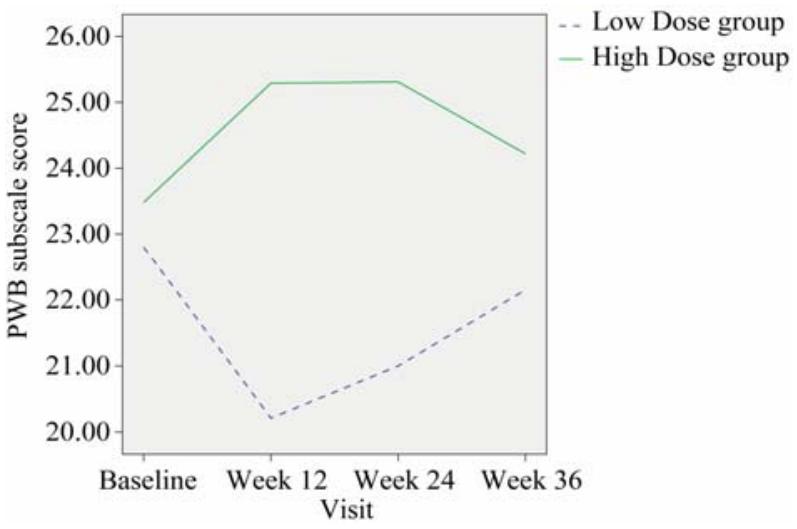

Figure 3. Change in the group mean of PWB subscale score.

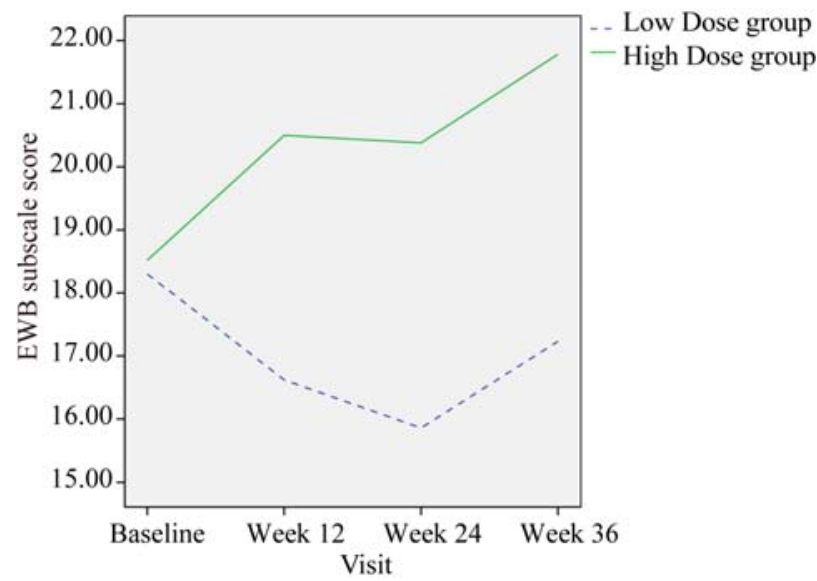

Figure 4. Change in the group mean of EWB subscale score. 
Table 8. Functional well-being (FWB) scale score and percent change (\%) from baseline

\begin{tabular}{lllll}
\hline \multirow{2}{*}{ Group } & \multicolumn{2}{c}{ Treatment } & \multicolumn{2}{c}{ Follow-up } \\
\cline { 2 - 5 } & Baseline & Week 12 & Week 24 & Week 36 \\
\hline Low dose & $18.85 \pm 4.77$ & $15.38 \pm 6.06(-12.59)$ & $16.93 \pm 5.62(-4.96)$ & $16.85 \pm 4.67(-7.38)$ \\
High dose & $17.43 \pm 5.23$ & $17.84 \pm 4.50(1.14)$ & $18.23 \pm 6.37(-4.50)$ & $19.22 \pm 5.38(-2.11)$ \\
${ }^{*}$ P value & 0.370 & 0.241 & 0.578 & 0.283 \\
\hline
\end{tabular}

${ }^{*} \mathrm{P}$ values were calculated based on two-sample t tests comparing differences in between-group means.

Table 9. FACT-G Total score and percent change (\%) from baseline

\begin{tabular}{lllll}
\hline \multirow{2}{*}{ Group } & \multicolumn{2}{c}{ Treatment } \\
\cline { 2 - 5 } & Baseline & Week 12 (\%) & Week 24 (\%) & Wollow-up \\
\hline Low dose & $80.37 \pm 14.24$ & $72.81 \pm 12.12(-3.98)$ & $73.98 \pm 13.92(-4.80)$ & $77.72 \pm 13.57(-1.25)$ \\
High dose & $80.43 \pm 14.39$ & $85.29 \pm 11.81(5.21)$ & $84.92 \pm 13.80(1.90)$ & $86.59 \pm 15.71(1.80)$ \\
${ }^{*}$ P value & 0.989 & $\mathbf{0 . 0 1 2}$ & $\mathbf{0 . 0 5 1}$ & 0.172 \\
\hline
\end{tabular}

${ }^{*} \mathrm{P}$ values were calculated based on two-sample t tests comparing differences in between-group means.

FACT-G Total score was significantly improvement after 3-month treatment $(\mathrm{p}=0.012)$ and 6-month treatment $(\mathrm{p}=0.051)$ in high dose group compared with that of in low dose group (Table 9, Figure 5).

The liver and renal functions had no significant changes before and after the clinical trial both in the High and the Low dose groups (data not shown).

\section{Discussions}

Quality of Life (QOL) assessment in cancer clinical research study has three major functions: 1) Evaluation of the symptom and therapeutic effects for the choice of optimal therapeutic methods; 2) Assessment of the effects of anti-cancer drugs, analgesics and antiemetic drugs; and 3) Evaluation of the long-term survival status of cancer patients after treatment [23].

The current evaluation system for cancer treatment is still using tumor response and survival as the main index. To completely kill the tumor cells is the aim of modern medicine for cancer treatment. So far the method of cancer treatment is still surgery, radiotherapy, and chemotherapy. There is no breakthrough yet in cancer treatment,

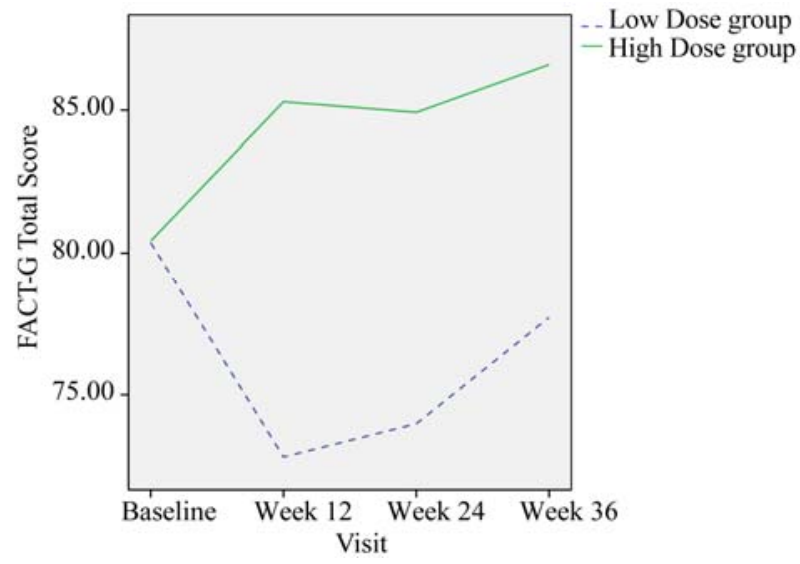

Figure 5. Change in the group mean of FACT-G total score. it is difficult to completely kill the tumor cells and reach the status of "tumor-free survival". The purpose of eradication of the tumor is very hard to reach; besides it may increase side effects and cause serious complications, increase the patient's suffering or even shorten the patient's survival.

In 1984, Schipper mentioned that effective treatment does not need to have complete regression of tumors, the body's reaction to the treatment is most important [24]. China's oncologist Sun Yan also said that people no longer content to cure the cancer but live a miserable life maimed or with functional disturbance [25].

The development of medicine should more concern the patient as a whole, rather than just concern the disease. TCM is holistic approach, and its overall effect should be evaluated comprehensively. Quality of life used to evaluate TCM efficacy is quite appropriate. The efficacy of Chinese medicine in cancer therapy is different from western medicine that is established in directly killing the tumor cells. Clinically patients' symptom relief and physical improvement seem more important. Through stabilizing the tumor and relieving symptoms, patients can have long-term survival with the tumor and avoid deterioration of quality of life. The main feature of Chinese medicine is not directly making the tumor shrink, but to improve patient physical and symptoms, the patients' survival may be prolonged. It is quite different from modern medical therapy, in which the tumor reduced in the short term, but the physical and quality of life decreased. Previous studies have confirmed that individuals who have lower baseline HRQOL scores have shorter survival [26]. Therefore, an important therapeutic goal for cancer patients with poor prognosis, to improve health-related quality of life (HRQOL) is a challenge for their health care providers. The Functional Assessment of Cancer Therapy-General (FACT-G) is used to assess HRQOL. In the approval of oncology drugs, the United States (US) Food and Drug Administration (FDA) and 
European Agency for the Evaluation of Medicinal Products consider HRQOL an important end-point [27].

Traditional Chinese Medicine (TCM) has a long history but its efficacy is not well-documented. Evidence of efficacy has to come from clinical trials. The design and conduction of clinical trials of TCM should follow the rigorous requirements of evidence-based medicine. Not many high quality controlled trials of traditional Chinese medicine have been done [28]. The common methodology of random selection, blinding and placebo control, followed by statistical analysis, should be followed when conducting TCM clinical trial [29]. Randomized controlled clinical trials have become the gold standard for evaluating the efficacy of a drug for disease treatment including herbal products. A well-designed and conducted clinical trial is very important for obtaining a reliable data to support efficacy and safety.

For most advanced cancer patients, completely eliminating the cancer cell is not the main therapeutic purpose. The study found that patient with advanced lung cancer treated with Chinese medicine the quality of life was significantly improved. The difference in FACT-G total score between the high dose and the low dose groups at 3-month and 6-month after treatment were statistically significant ( $\mathrm{p}=0.012$ and 0.051 respectively). In subscale scores, PWB and EWB scores were also significantly improved after treatment. We believe that the study herbal medicine could be beneficial to lung cancer patients.

Drug compliance may be considered as a specific outcome measure in clinical trials. This study shows that the compliance of the high dose group was significantly higher than the low dose group, which indirectly indicated the proper dosage of study medicine may improve the quality of life of patients with lung cancer help through keeping the confidence of drug taking. Claims of enhanced compliance could be supported by evidence of clinical benefit [30]. In this study, the clinical benefit in the high dose group was supported by its higher compliance.

\section{Conclusions}

Efficacy of Traditional Chinese Medicine (TCM) can be put on well-designed randomized controlled clinical trials (RCT). Conventional outcome criteria may not be applicable. Holistic approach using quality of life to evaluate the efficacy of TCM is an important compromise. The study demonstrated that quality of life of patients with lung cancer was significantly improved.

\section{Acknowledgements}

The authors would like to acknowledge the Ming Lai
Foundation for the support given to the Institute of Chinese Medicine, CUHK.

The authors report no conflicts of interest.

\section{REFERENCES}

[1] Hong Kong Cancer Registry, "Cancer Incidence and Mortality in Hong Kong 1998-1999,” Hong Kong Authority, Hong Kong, 2000.

[2] X. S. Wang, L. J. Di, C. C. Reyes-Gibby, H. Guo, S. J. Liu and C. S. Cleeland, "End-of-Life Care in Urban Areas of China: A Survey of 60 Oncology Clinicians,” Journal of Pain and Symptom Management, Vol. 27, No. 2, 2004, pp. 125-132. doi:10.1016/j.jpainsymman.2003.06.002

[3] K. Chen, P. P. Wang, B. C. Sun, et al., “Twenty-Year Secular Changes in Sex Specific Lung Cancer Incidence Rates in an Urban Chinese Population," Lung Cancer, Vol. 51, No. 1, 2006, pp. 13-19. doi:10.1016/j.lungcan.2005.08.013

[4] L. D. Li, F. Z. Lu, S. W. Zhang, R. Mu, X. D. Sun, X. M. Huangpu, J. Sun, Y. S. Zhou, N. H. Ouyang, K. Q. Rao, Y. D. Chen and A. M. Sun, "Analysis of Cancer Mortality and Distribution in China from Year 1990 through 1992: an Epidemiologic Study," Chinese Journal of Oncology, Vol. 18, No. 6, 1996, pp. 403-405.

[5] Y. S. Zhou, S. W. Zhang and L. D. Li, "Analysis for Death for All and Malignant Tumors for China Population,” Bull China Can, Vol. 6, 1997, pp. 9-11.

[6] National Office for Cancer Prevention and Control (NOCPC) of the Chinese Ministry of Health Investigate for Malignant Tumor Mortality in China, People's Health Publishing House, Beijing, 1979.

[7] L. D. Li, F. Z. Lu, S. W. Zhang, et al., "Analysis of Variation Trend and Short-Term Detection of Chinese Malignant Tumor Mortality during Twenty Years," Chinese Journal of Oncology, Vol. 19, No. 1, 1997, pp. 3-9.

[8] T. K. Yun and S. Y. Choi, "Non-Organ Specific Cancer Prevention of Ginseng: A Prospective Study in Korea," International Journal of Epidemiology, Vol. 27, No. 3, 1998, pp. 359-364. doi:10.1093/ije/27.3.359

[9] B. H. Lao, H. C. Ruckle and T. Botolazzo, "Chinese Medicinal Herbs Inhibit Growth of Murine Renal Cell Carcinoma,” Cancer Biother, Vol. 9, No. 2, 1994, pp. 153161. doi:10.1089/cbr.1994.9.153

[10] J. Z. Wang, H. Tsumara and K. Shimura, “Antitumor Activity of Polysaccharide from Chinese Medicinal Herb, Acanthopanax Giraldii Harms,” Cancer Letters, Vol. 65, No. 1, 1992, pp. 79-84.

doi:10.1016/0304-3835(92)90216-I

[11] T. Tode, Y. Kikuchi and T. Kita, "Inhibitory Effects by Oral Administration of Ginsenoside Rh2 on the Growth of Human Ovarian Cancer Cells in Nude Mice,” Journal of Cancer Research and Clinical Oncology, Vol. 120, No. 1-2, 1993, pp. 24-26. doi:10.1007/BF01200720

[12] J. K. Liu, "Prospect of the Development of New Natural Drugs as Foreshadowed by the R \& D of the World 20 
Leading Pharmaceutical Corporations," Chinese Traditional and Herbal Drugs, Vol. 31, No. 7, 2000, pp. 481-487.

[13] L. A. Ries, C. L. Kosary and B. F. Hankey, "Lung and Bronchus Cancer: SEER Cancer Statistics Review, 1973-1996,” National Cancer Institute, Bethesda, MD, 1999.

[14] Z. Rosenberg, "Treating the Undesirable Effects of Radiation and Chemotherapy with Chinese Medicine," Journal of Chinese Medicine, Vol. 55, No. 55, September 1997, pp. 29-30.

[15] S. Y. Lin, L. M. Liu, L. C. Wu, "Effects of Shenmai Injection on Immune Function in Stomach Cancer Patients after Chemotherapy," Chinese Journal of Integrated Traditional and Western Medicine, Vol. 15, No. 4, 1995, pp. 451-453.

[16] H. Boon, J. B. Brown, A. Gavin, M. A. Kennard and M. Stewart, "Breast Cancer Survivors' Perceptions of Complementary/Alternative Medicine (CAM): Making the Decision to Use or Not to Use," Qualitative Health Research, Vol. 9, No. 5, 1999, pp. 639-653. doi:10.1177/104973299129122135

[17] M. A. Richardson, T. Sanders, J. L. Palmer, A. Greisinger and S. E. Singletary, "Complementary/Alternative Medicine Use in a Comprehensive Cancer Center and the Implications for Oncology,” Journal of Clinical Oncology, Vol. 18, No. 13, 2000, pp. 2505-2514.

[18] W. XU, A. D. TOWERS, P. LI and J.-P. Collet, "Traditional Chinese Medicine in Cancer Care: Perspectives and Experiences of Patients and Professionals in China," European Journal of Cancer Care, Vol. 15, No. 4, September 2006, pp. 397-403. doi:10.1111/j.1365-2354.2006.00685.X

[19] J. L. Tang, S. Y. Zhan and E. Ernst, "Review of Randomised Controlled Trials of Traditional Chinese Medicine," British Medical Journal, No. 319, No. 7203, 1999, pp. 160-161.

[20] K. Linde, W. B. Jonas, D. Melchart and S. Willich, "The Methodological Quality of Randomized Controlled Trials of Homeopathy, Herbal Medicines and Acupuncture," International Journal of Epidemiology, Vol. 30, No. 3,
2001, pp. 526-531. doi:10.1093/ije/30.3.526

[21] L. Xu, J. Li, M. M. Zhang, C. L. Ai and L. Wang, "Chinese Authors Do Need CONSORT: Reporting Quality Assessment for Five Leading Chinese Medical Journals," Contemporary Clinical Trials, Vol. 29, No. 5, 2008, pp. 727-731. doi:10.1016/j.cct.2008.05.003

[22] I. D. Giovanna and G. Hayes, "Principles of Clinical Research," Wrightson Biomedical Publishing Ltd., UK, 2001, p. 106.

[23] L. Jian and C. Da-tong, "Evaluation of the Quality of Life in the Treatment of Malignant Tumor," Chinese Journal of Oncology, Vol. 24, No. 4, 2002, pp. 411-413.

[24] H. Schipper, J. Clinch, A. McMurray, et al., "Measuring the Quality of Life of Cancer Patients: The Functional Living Index-Cancer-Development and Validation," Journal of Clinical Oncology, Vol. 2, No. 5, 1984, pp. 472-483.

[25] T. Z. Zhang and G. W. Xu, “Oncology,” Tianjin Science and Technology Press, Tianjin, 1996, p. 830.

[26] M. Dharma-Wardene, H.-J. Au, J. Hanson, D. Dupere, J. Hewitt and D. Feeny, "Baseline FACT-G Score is a Predictor of Survival for Advanced Lung Cancer," Quality of Life Research, Vol. 13, No. 7, 2004, pp. 1209-1216. doi:10.1023/B:QURE.0000037481.36604.eb

[27] K. Gondek, P. P. Sagnier, K. Gilchrist and J. M. Woolley, "Current Status of Patient-Report Outcomes in Industry-Sponsored Oncology Clinical Trials and Product Labels,” Journal of Clinical Oncology, Vol. 25, No. 32, 2007, pp. 5087-5093. doi:10.1200/JCO.2007.11.3845

[28] J. L. Tang, S. Y. Zhan and E. Ernst, "Review of Randomised Controlled Trials of Traditional Chinese Medicine," British Medical Journal, Vol. 319, No. 7203, 1999, pp. 160-161.

[29] P.-Ch. Leung, "A Practical Way of Research in Chinese Medicine," Annals of the Academy of Medicine of Singapore, Vol. 35, No. 11, 2006, pp. 770-772.

[30] M. Cavaille, "Coll Impact of Compliance on Drug Development: An FDA Perspective," Transplantation Proceedings, Vol. 31, 1999, pp. 40S-41S. 